\title{
Review of: "Seaweed cellulose scaffolds derived from green macroalgae for tissue engineering"
}

\author{
Natalia Vapniarsky \\ Potential competing interests: The author(s) declared that no potential competing interests exist.
}

Manuscript by Nurit Bar-Shai et al., reports a method for decellularization and manufacturing of cellulose scaffolds from two types of macroalgae. The authors further evaluate the biocompatibility of these scaffolds for supporting the growth of fibroblasts. The study is well structured and the manuscript is well written, however some additional important aspects could be addressed to make this work more relevant for tissue engineering field.

Besides the advantage of cheaper manufacturing process and high growth rate, the benefits of using the macroalga-derived cellulose matrixes are not emphasized. It would be important to compare Bar-Shai's cellulose matrix to other cellulose matrixes in terms of cell growth, biodegradability, mechanical properties, porosity, permeability, compatibility with growth of other cell types besides fibroblasts, discuss potential tissues that would be possible to manufacture using these scaffolds. In other words, discussion and testing of more targeted applications of macroalga-derived cellulose matrixes would greatly benefit this work.

Cytotoxicity was only partially addressed by the authors. The media extracts were obtained upon $24 \mathrm{~h}$ of soaking the scaffolds in culture media. Twenty-four hours is very limited time to assess the potential damaging effects of scaffold's degradation products, and, again, the comparison to other commercially available cellulose matrixes is lacking.

Although authors mention the ECM deposition aspects in the discussion's future directions, ECM deposition could be assessed and reported in this study. For example, simple histology of the recellularized scaffolds could have been performed at various time points and ECM deposition could be assessed with help of special stains. (Picrisirius red or others).

Recommendation: Accept for publication with minor corrections. 